\title{
Analysis of Clinical Percussion Signals Using Matching Pursuit
}

\author{
Jeffery Dech¹, Moinuddin Bhuiyan*1,2, Roman Gr. Maev¹,2 \\ ${ }^{1}$ Department of Physics, University of Windsor, 401 Sunset Ave., Windsor, Ontario N9B 3P4, Canada. \\ ${ }^{2}$ Institute for Diagnostic Imaging Research, University of Windsor, 401 Sunset Avenue, Windsor Ontario \\ N9B 3P4 Canada.
}

* Corresponding author: Tel. 1-519-977-7440; email: moni.bit.ctg@gmail.com

Manuscript submitted June 22, 2014; accepted April 7, 2015.

doi: 10.17706/ijcee.2015.7.4.248-260

\begin{abstract}
Clinical percussion is a method of eliciting sounds from the body by tapping with either a percussion hammer or fingers to determine the area under the perused is air filled, fluid filled, or solid, and is used in clinical examinations to assess the condition of the thorax or abdomen. Successful diagnosis today is still highly subjective and dependent's on physician skill, experience and require quite surrounding areas. An automated system capable of delivering standardized percussion analysis would remove these limitations on the technique and allow for its usage by those without such specialized training and years of necessary experience. For this to be possible, efficient and informative signal processing algorithms must be employed. In this investigation, clinical percussions from healthy volunteers taken by trained medical professionals were analysed via the matching pursuit (MP) algorithm. Various types of possible dictionaries are discussed comparing their efficiency and convergence behaviour. Noise filtering methods are discussed and a noise reduction method based on MP analysis results is presented.MP is also compared to other methods for representing clinical percussions with regards to informativeness and efficiency. MP is $\mathcal{O}(n \log n)$ which is more efficient than current methods which have a complexity of at least $\mathcal{O}\left(n^{3}\right)$.
\end{abstract}

Key words: Digital signal processing, medical percussion, matching pursuit, pneumothorax.

\section{Introduction}

Clinical percussion is the method of eliciting sounds by tapping different areas of the human body either with finger tips or a percussion hammer [1], [2]. By interpreting the audible percussion sounds, a skilled physician can determine the location and dimensions of the underlying body organs in norm and pathology as well as find out whether the underlying tissues contain air or fluid inclusions, density anomalies or anatomical defects [1], [3], [4]. Percussion has been successfully used for the diagnosis of such potentially lethal conditions as traumatic tension in the pneumothorax. The success of this classical method depends on the experience and individual talent of the physician whose trained ears and brain learn to interpret subtle differences between percussion sounds from many patients in a quiet room. Overall, these factors lead to a method that while proven to be diagnostically informative, is subjective and has low sensitivity in comparison to other diagnostic methods. In this respect, automated analysis and computerized representation of percussion findings might help determine the pathological condition in a more objective and standardized way. The use of digital signal processing techniques to extract diagnostic information from percussion sounds would comprise a major step towards advancing the utility of medical percussion 
beyond its traditional scope. The excitation and sensing modules can be implemented in a hand-held percussion device equipped with an embedded microcomputer to perform data acquisition and classification of the signals in a standardized and reliable manner. Such portability would enable rapid on-site diagnostics of pulmonary trauma in situations where immediate or thorough clinical examination be a physician would be impossible. The majority of the preceding work attempting to objectively classify percussion sounds is based on Fourier analysis methods [5]. Previous investigations by our group [6]-[8] have shown that medical percussion signals are well suited to decomposition into a sum of exponentially damped sinusoids (EDS). Non-linear spectral fitting procedures have been able to approximate various percussions using 1-3 damped harmonics. Unfortunately, these types of fits are difficult to implement with automated procedures due to the complexity of the method and the requirement for semi-empirical fitting parameters to be used [6]. The Matrix Pencil Method (MPM) has also be shown to be a method capable of decomposing percussions into a sum of EDS components that is much more resilient to noise than spectral fitting procedures and is an easily automated algorithm [7]. The MPM minimizes the norm of all possible solutions through singular value decomposition (SVD). This results in a least-squares optimal solution [8] but this also tends to spread the signal energy across EDS modes which results in fits with a large number of small order terms [9]. In addition, for $m \times n$ matrix, SVD calculations are $O\left(m n^{2}\right)$ [10] which, combined with the eigenvalue calculation required to determine system poles, could result in a computationally expensive solution that may not be desirable for real time application. Many signal decomposition methods such as wavelet and Fourier analysis attempt to project a signal onto a basis, often orthogonal, that provides for sparse decompositions and ideally a representation that well models the nature of the problem. However, modelling an arbitrary signal with a given basis may have serious drawbacks if the basis is not well suited to that specific signal. In order to overcome these issues, an over-complete basis characterized by basis components representing a variety of signal behaviours can be used [9], [11]. Decompositions using over-complete basis sets (i,e. non-orthogonal) can be carried out using various methods; these include the method of frames [12], "best basis" methods that attempt to determine an appropriate basis through the use of metrics such as entropy [13] or other algorithms [14], basis pursuit [15], and matching pursuit algorithms [11], [16]-[18]. Matching pursuit (MP) is a method of decomposing signals using time-frequency dictionaries [9], [11] with applications to parametric audio coding and classification [9], [19], audio and video compression [20] and de-noising [21]. The goal of this method is to iteratively determine the best signal decomposition from an over complete atomic dictionary. Typical dictionaries are comprised of symmetric Gabor atoms [9], [11] and other windowed harmonic atoms [9], [21] or wavelet basis dictionaries that are chosen based on their similarity to the behaviour of the signals being analysed. Applying MP decomposition to medical percussions signals can quickly determine an atomic decomposition of the signal and by analyzing critical parameter; the signals can be grouped into subclasses. Historically, the clinical study of percussions identifies two signal groups known as "tympanic" and "resonant" and the ability to assign a signal to one of the two groups opens the possibility for automated diagnostic of air or liquid inclusions in the thorax or abdomen.

\section{Materials and Methods}

\subsection{Materials}

The percussions analysed in this study were collected on healthy volunteers at the Detroit Medical Center by trained medical professionals. The testing of human subjects was performed according to the protocol \# 0710005340 ("Portable Pulmonary Injury Device") approved by the human investigation committee for the Wayne State University institutional Review Board (M1) for the period of 09/25/2008 through $09 / 24 / 2009$. 


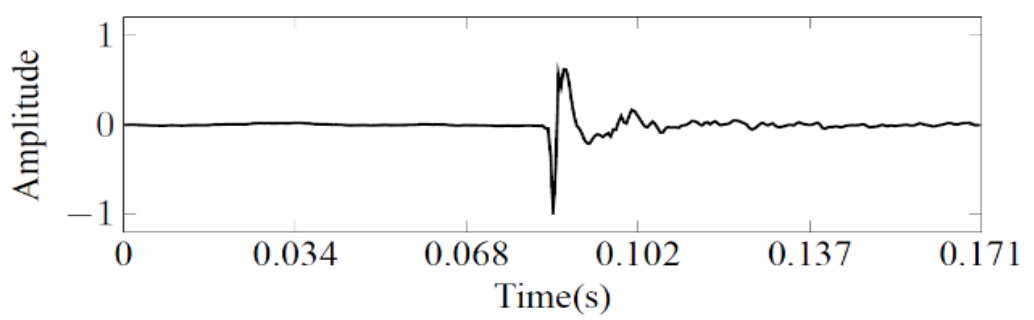

(a)

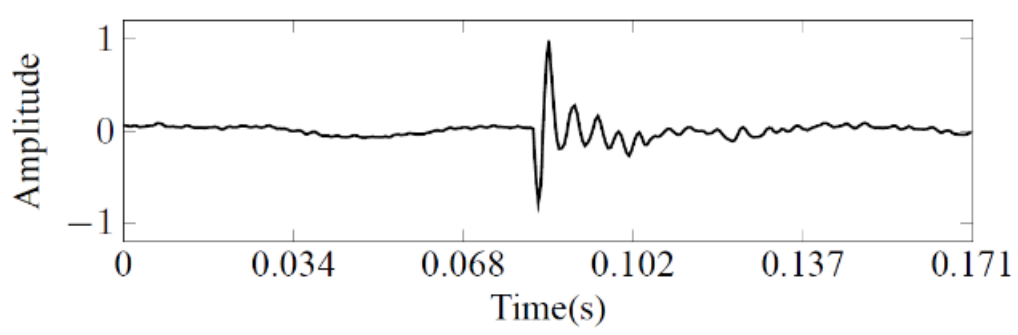

(b)

Fig. 1. Two examples of audible percussion signals collected from healthy volunteers: (a). Signal from the left sub clavicular area ("resonant" character); (b). Signal from the left abdominal area ("tympanic" character).

A mediator plate (plessimeter) was placed on the volunteer's chest or abdomen and was struck by a neurological hammer to produce a percussion signal. Percussions were received with a tripod-based omnidirectional electrical condenser air microphone. For all signals, the microphone was placed at a distance of $150-300 \mathrm{~mm}$ from the percussion site. The signals were then amplified and digitized at a $48 \mathrm{kHz}$ using a 24-bit computer sound card. Two examples of percussion waveforms can be seen in Fig. 1. The "resonant" percussion (Fig. 1(a)) is a typical percussive response from normal lung tissue in the area of the upper chest in the sub clavicular area while the "tympanic" signal (Fig. 1(b)) is typically expected in the case of pneumothorax conditions [1]. It is worth noting that the signals measured in these studies are similar in appearance to those found by Murray and Neilson [5].

\subsection{Methods}

\subsubsection{Matching pursuit}

Matching pursuit is an iterative, greedy algorithm for decomposing signals in terms of a series of basis functions chosen from a over-complete dictionary [11]. This algorithms iteratively finds best matches of a signal to vectors in usually highly redundant and over-complete set of time-limited functions, or atoms, called a dictionary [11]. Using a dictionary that is comprised of elements that resemble the underling signal structures, a sparse signal representation can be formed via MP. First, a dictionary of functions $g_{m} \in D$ is defined with $g_{m} \in H$, a Hilbert space. The goal of the pursuit is to determine an M term expansion of a signal $x[n]$ such that

$$
x[n]=\sum_{i=1}^{M} \alpha_{i} g_{i}[n]
$$

With each term corresponding to a weight $\alpha_{i} \in \mathcal{R}$ and a dictionary function $g_{i} \in D$. At every iteration $i$, the atom selected is the one that minimizes the energy of the residual

$$
r_{i+1}=\left\{\begin{array}{cc}
x[n]-\alpha_{i} g_{i} & \text { if } i=0 \\
r_{i}-\alpha_{i} g_{i} & \text { if } i>0
\end{array}\right.
$$


Provided that $\left\|g_{m}\right\|^{2}=1$ for $g_{m} \in D$

$$
\begin{gathered}
g_{i}=\underset{g_{m} \in D}{\operatorname{argmax}}\left\langle g_{m} \mid r_{i}\right\rangle \\
\alpha_{i}=\left\langle g_{i} \mid r_{i}\right\rangle
\end{gathered}
$$

where $\left\langle g_{i} \mid r_{i}\right\rangle$ is the inner product,

$$
\left\langle g_{i} \mid r_{i}\right\rangle=\sum_{n=1}^{N} g_{i}[n] . r_{i}[n]
$$

The algorithm will continue for $M$ iterations giving

$$
x[n] \approx \sum_{i}^{M} \alpha_{i} g_{i}=\sum_{i=1}^{M}\left\langle g_{i} \mid r_{i}\right\rangle g_{i}
$$

The number of iterations carried out is typically determined by predefined stopping criteria dependent on underlying signal characteristics. Typical criterion include producing an expansion of a set number of iterations or continuing until the residual after the $M-t h$ residual $r_{M}$ has an $L_{2}$ norm that falls below a certain fraction of the original signal energy [11]. In the latter case, if iterations are stopped after a fraction of the original energy $\in$ remains, the criterion would be

$$
\frac{\left\|r_{M}\right\|^{2}}{\|x\|^{2}}<\epsilon
$$

This value is arbitrary and is determined by the application.

\subsubsection{Atomic dictionaries}

Due to the general nature of the MP algorithm, it is important to select a basis dictionary that has a similar structure as the signals in question in order to achieve sparsity. On the other hand, performing pursuits over large dictionaries can be computationally expensive which makes choosing an optimal, easily tractable dictionary important. Since the only requirement is that an atom $g_{i} \in H$, many types of function dictionaries are available. The nature of medical percussions presents a few obvious initial choices. The harmonic nature of percussive signals has been analysed in previous studies [6]-[8] and EDS decompositions have been used in the past to exploit this.

The tympanic signal can typically be well fitted with a single Lorentzian curve in Fourier space. The resonant signal however is more broadband requiring a larger number of terms in the fits. Both signal types are band limited to approximately $1 \mathrm{KHz}$. The signals have been characterized using a sum of a few damped sinusoidal oscillators and it has been found the signals can be characterized using one to three of these atoms in summation. In addition, differences can be seen between the Fourier spectra of the two classes of percussions (Fig. 2).

However, pursuits involving purely EDS dictionaries exhibit behaviours that can potentially introduce artefacts into the residual that result in the addition of energy rather than its removal [22]. Additional dictionary elements need to be included with a higher level of localization in order to ensure that convergence can be achieved. This will require additional computation to pursue over a larger dictionary which introduces further computational complexity rather than reducing it. Gabor dictionaries [11], [23] consist of Gaussian windows modulated by sinusoidal oscillators and are often used in MP decompositions [11], [24]. These atoms are defined in terms of a parameter set $\left(t_{c}, t_{w}, f, \varnothing\right)$ giving the time center of the Gaussian window $t_{c}$ the window size $t_{w}$, and the carrier wave's frequency $f$ and phase $\emptyset$. 


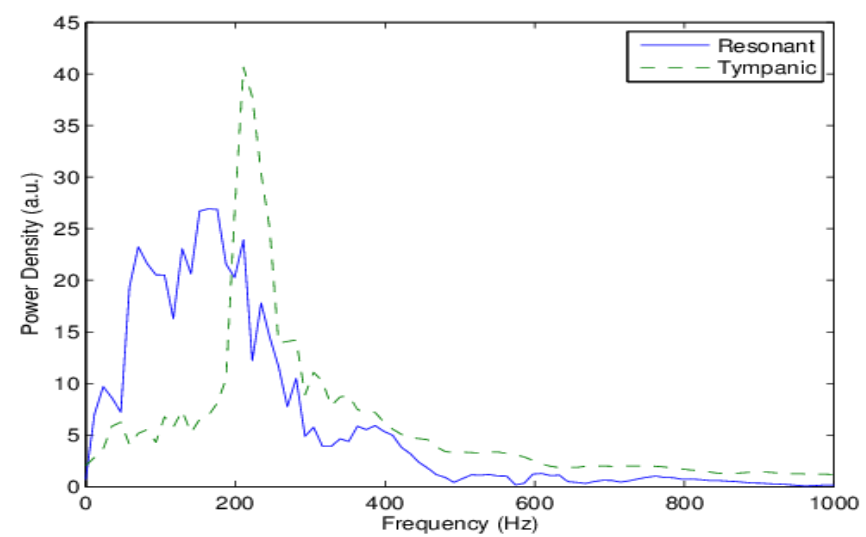

Fig. 2. Power spectrum of a typical tympanic signal (dashed) and resonant signal (solid) percussion.

\section{Implementation}

The experimental setup used for capturing the medical percussions was configured to begin recording audio 30ms before the actual signal event occurs. This window (or "pre-stage") allows for recording of the ambient background noise that can be used for noise filtering in the signal processing stage. This pre-listen waveform was assumed to be representative of the noise during the percussion event. Prior to MP analysis, signals were processed with noise reduction filter constructed using acoustic information gathered in the pre-record window.

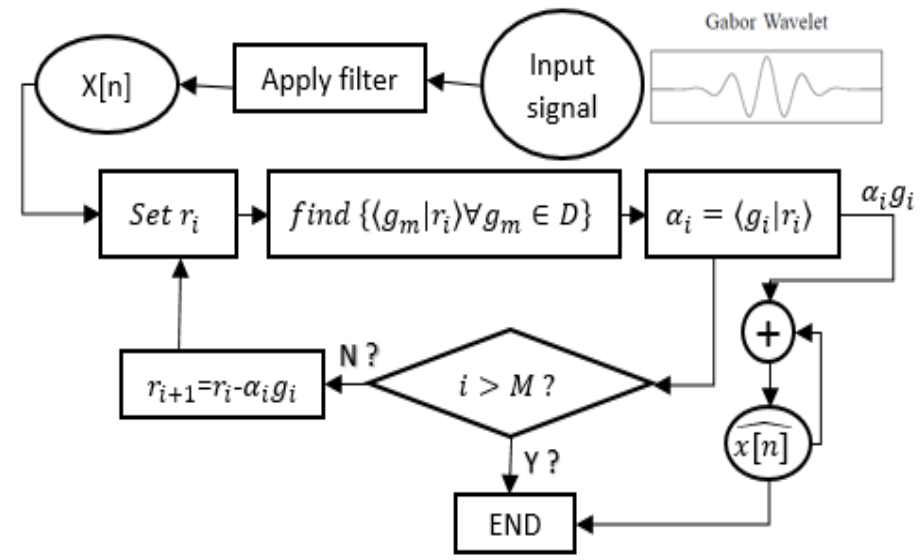

Fig. 3. Block diagram of the matching pursuit algorithm.

Signals were first filtered using the method of outlined in Section 3.1. The cleaned signal was then analyzed using MPTK, setting it as the initial residual. At each iteration, the inner product set is determined; the atom is chosen and subtracted from the residual. The process continues for 25 iterations.

These cleaned signals were then used as the input signal for the MP algorithm with no additional cropping or filtering. Pursuits were carried out using the Matching Pursuit Toolkit (MPTK) [24]. A block diagram of the algorithm can be seen in Fig. 3.

\subsection{Pre-filtering}

The noise reduction scheme is outlined in [25]. Since the percussion signals exhibit a harmonic structure, a filter preserving the underlying harmonics would be ideal in a noise filtering method. We assume the usual model of a signal 


$$
x(t)=s(t)+n(t)
$$

where, $s(t)$ and $n(t)$ are the percussion and noise signal, respectively. If we have $S(p, k), N(p, k)$ and $X(p, k)$ as the $k-t h$ spectral elements of the windowed frame $p$ of the percussion, noise and measured signal respectively, we construct a filter using a gain $G(p, k)$ for a given component to determine the percussion spectrum estimate

$$
S^{\wedge}(p, k)=G(p, k) X(p, k)
$$

The pre-listening stage was assumed to be approximate to $n(t)$ for the purpose of determining the frequency spectrum $|N(p, k)|^{2}$. A local SNR estimate $\operatorname{SNR}^{\wedge}(p, k)$ is then determined and a Wiener filter is constructed as

$$
G(p, k)=\frac{\operatorname{SNR}^{\wedge}(p, k)}{1+S N R^{\wedge}(p, k)}
$$

Giving, the signal estimate $S(p, k)$ as in (9).

\subsection{Matching Pursuit}

Pursuits were performed using MPTK with a stopping criterion of 25 iterations. MPTK is an open software library that provides a fast MP implementation for a variety of atomic function classes. Pursuits are calculated in terms of short-time Fourier transforms which allow for an $O(n \log n)$ implementation of the algorithm as opposed to the general $O\left(n^{2}\right)$.

A dictionary was generated consisting of Gabor atoms with Gaussian window sizes of $2^{5}$ to $2^{13}$ samples which correspond to times of 0.6 and $85 \mathrm{~ms}$ respectively. All atoms were evenly spaced $0.6 \mathrm{~ms}$ along the time axis with a frequency resolution of $\pm 0.75 \mathrm{~Hz}$. To give an idea of scale, percussions used in this analysis have durations of less than $40 \mathrm{~ms}$ and typically greater than $10 \mathrm{~ms}$. For testing, a sample database of 190 clinical percussion scans was assembled with an approximately equal number of tympanic and resonant percussions included in the dataset.

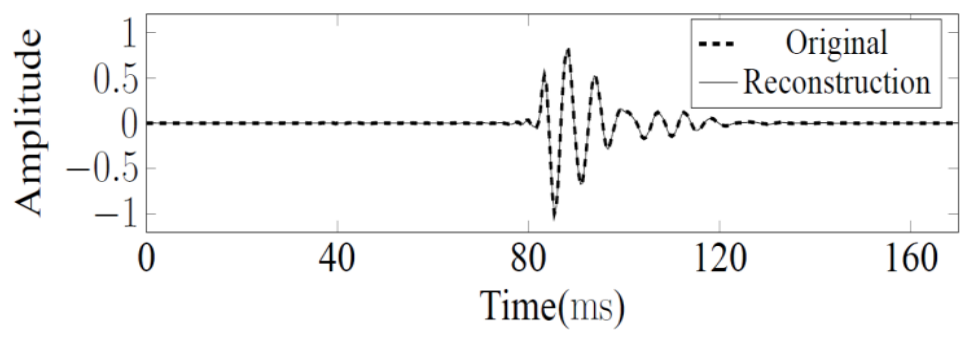

Fig. 4. Matching pursuit reconstruction of a tympanic signal superimposed over the original signal.

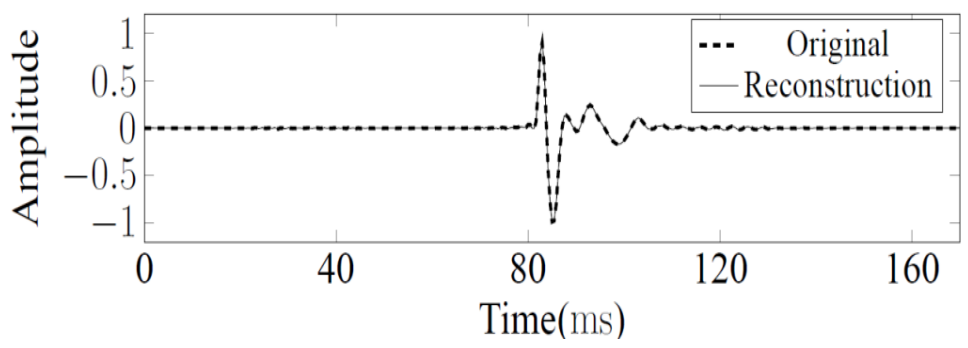

Fig. 5. Matching pursuit reconstruction of a resonant signal overlaying the original percussion signal. 


\section{Results and Analysis}

Fig. 4 and Fig. 5 show the time series MP reconstructions of a resonant and tympanic percussion respectively. The corresponding parameter values for the tympanic and resonant percussions can be seen in Tables 1 and 2 respectively. Pursuits will produce a greedy expansion resulting in the most significant information being contained within the first few atoms considering that percussion signals are well localized due to their rapid decay. The impulsive nature of the signals causes the largest amplitude atom to align with the onset of the signal.

Table 1. Pursuit Atom Parameters for a Typical Tympanic Percussion Signal

\begin{tabular}{|l|l|l|l|l|}
\hline Amplitude [A.U] & $T_{c}(\mathrm{~ms})$ & $F_{c}(\mathrm{~Hz})$ & $T_{w}(\mathrm{~ms})$ & $\Phi(\mathrm{rad})$ \\
\hline 14.94 & 753.3 & 197.0 & 213.3 & -0.01 \\
\hline 2.85 & 726.7 & 350.8 & 213.3 & -1.73 \\
\hline 2.31 & 92.0 & 178.7 & 213.3 & -0.82 \\
\hline 2.16 & 65.3 & 68.8 & 106.7 & 2.95 \\
\hline 1.79 & 88.0 & 276.9 & 106.7 & 0.77 \\
\hline
\end{tabular}

Table 2. Pursuit Atom Parameters for a Typical Resonant Percussion Signal

\begin{tabular}{|c|c|c|c|c|}
\hline Amplitude [A.U] & $T_{c}(\mathrm{~ms})$ & $F_{c}(\mathrm{~Hz})$ & $T_{w}(\mathrm{~ms})$ & $\Phi(\mathrm{rad})$ \\
\hline 16.57 & 79.3 & 136.2 & 106.7 & 0.57 \\
\hline 3.08 & 74 & 344.2 & 213.3 & 2.80 \\
\hline 1.86 & 88.7 & 113.5 & 213.3 & -1.43 \\
\hline 1.25 & 88.3 & 43.2 & 106.7 & 0.47 \\
\hline 1.17 & 64.0 & 156.0 & 42.7 & -3.05 \\
\hline
\end{tabular}

As the time centres of the atoms are parameters of the fit, information regarding the general location of the onset of the signal is provided by the pursuit. If an appropriate window is chosen, cropping and rough alignments of different signals can be performed without additional an algorithm which aids comparison between separate signals. This holds advantage over methods such as spectral fitting which are highly sensitive to time displacement and therefore require precise alignment of onset positions in order to compare different signals.

\subsection{Decomposition Behavior}

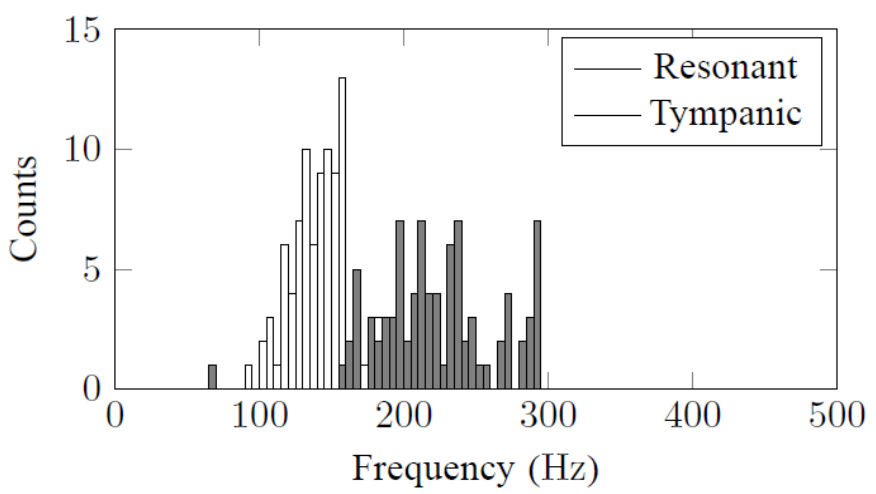

Fig. 6. Gabor frequencies for initial atomic match of tympanic (dark) and resonant (light) signals. Resonant signal will typically match the most significant atom around $150 \mathrm{~Hz}$ while tympanic decompositions will first match to an atom in the range of 200-300 Hz. 
The most significant (or primary) atom will be matched first in a greedy procedure. If we consider the distribution of the carrier frequency of this primary atom across the dataset as in Fig. 6, a clear grouping can be seen within both resonant and tympanic type signals. Resonant percussions tend to have a primary atom with a carrier frequency of around $150 \mathrm{~Hz}$ while tympanic signals tend to initially match to an atom in neighbourhood of 200-300Hz. Previous investigations [8] agree with these results. Using an EDS basis, resonant percussions were found to have strong primary peaks in the area of $100 \mathrm{~Hz}$ while tympanic matches usually occurred in the $200-300 \mathrm{~Hz}$ range. A direct comparison of results cannot be determined in this fashion due to the fact that highly localized nature of Gabor atoms will not produce a decomposition that can be directly compared with least-squared fitted EDS components fitted globally to the signal.

\subsection{Convergence}

The energy contained within the residual after every iteration can be measured and from this, the signal decay at iteration $i$ can be determined.

$$
D(i)=\frac{\left\|r_{i}\right\|^{2}}{\|x\|^{2}}
$$

The cut-off length can be defined as the number of iterations necessary for the deposited energy to fall below a given threshold $\Delta=0.5 \% \equiv 0.005$

$$
L_{c}=I \text {, when } D(I)-D(I-1)<\Delta
$$

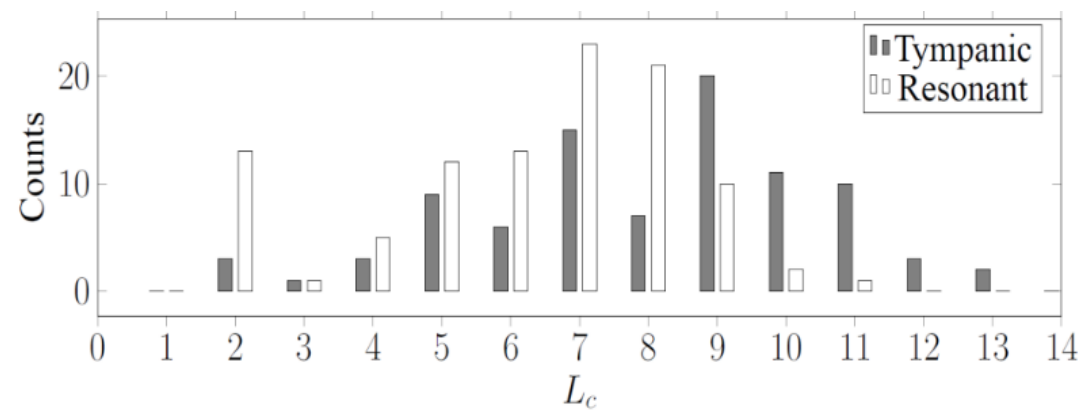

Fig. 7. Distribution of the number of iterations required for atoms to contribute less than $0.5 \%$ to the total energy. Resonant percussion (white) typically converge quicker than tympanic signals (black).

The distribution of these cut-off values is shown in Fig. 7. Resonant percussions generally have a more rapid convergence than tympanic signals which can be explained by their more localized energy distribution in the time domain. While all $L_{c}$ values are below 15, many of these low amplitude atoms will likely be unnecessary and uninformative with regards to signal classification. The signal decompositions outlined in Tables 1 and 2 show an over 80\% drop in amplitude after the first atomic match and at least a $95 \%$ drop by the tenth. Many of these low amplitude atoms will be essentially correction terms in the time domain. In this case, it is quite possible that only a few atomic matches are necessary in order to determine the nature of the percussion signal which would result in decreased computation and an increase in processing speed.

\subsection{Noise Reduction}

All signals were pre-filtered according to the method outlined in Section 3.1 in order to reduce external noise (Fig. 8). The noise signal $n(t)$ was then determined after filtering as per (8). Using this estimate, $s(t)$ and $n(t)$ were compared to determine the SNR. This value typically lies within the range of $5-20 \mathrm{~dB}$ as 
can be in Fig. 9. This variance can likely be explained by variations in the testing environment. Percussions were taken from multiple volunteers and there is no guarantee that the external environment remained constant throughout the duration of these tests.

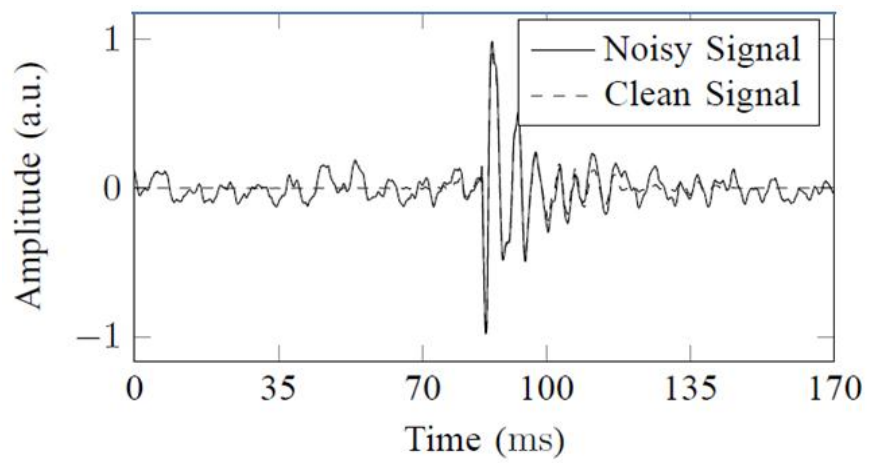

Fig. 8. Comparison of a noisy percussion signal and the same cleaned signal after de-noised.

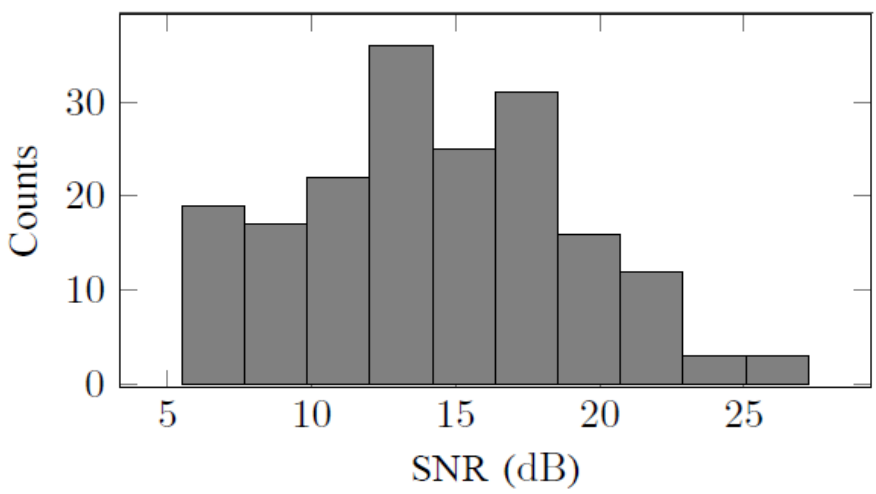

Fig. 9. SNR comparing cleaned percussion signals to extracted noise. Measurements vary across the database due to varying experimental acoustic environment.

Table 3. Atomic Parameters Corresponding to a Decomposition of the Signal Seen in Fig. 8 without the Application of Noise Reduction

\begin{tabular}{|c|c|c|c|c|}
\hline Amplitude [A.U] & $T_{c}(\mathrm{~ms})$ & $F_{c}(\mathrm{~Hz})$ & $T_{w}(\mathrm{~ms})$ & $\Phi(\mathrm{rad})$ \\
\hline 14.83 & 773.3 & 187.50 & 213.3 & 0.67 \\
\hline 6.30 & 800.0 & 409.42 & 106.7 & 1.95 \\
\hline 5.42 & 860.0 & 202.15 & 213.3 & -2.26 \\
\hline 4.03 & 66.7 & 125.98 & 853.3 & 0.20 \\
\hline 3.80 & 1026.7 & 169.99 & 213.3 & -2.92 \\
\hline
\end{tabular}

Table 4. Atomic Parameters Corresponding to a Decomposition of the Signal Seen in Fig. 8 after the Application of Noise Reduction Filters

\begin{tabular}{|c|c|c|c|c|}
\hline Amplitude [A.U] & $T_{c}(\mathrm{~ms})$ & $F_{c}(\mathrm{~Hz})$ & $T_{w}(\mathrm{~ms})$ & $\Phi(\mathrm{rad})$ \\
\hline 7.17 & 773.3 & 188.96 & 213.3 & 0.57 \\
\hline 3.14 & 800.0 & 410.16 & 106.7 & 1.92 \\
\hline 2.60 & 893.3 & 240.97 & 106.7 & 0.66 \\
\hline 1.91 & 840.0 & 222.66 & 426.7 & -1.04 \\
\hline 1.37 & 873.3 & 328.13 & 53.3 & 0.64 \\
\hline
\end{tabular}


The signal in Fig. 8 was analysed using the MP method both before and after noise filtering in order to determine how the MP decomposition would change in the presence of a noisy signal. Tables 3 and 4 compare the decompositions of the signal with and without noise respectively. When considering the first two significant atoms, they are of nearly identical time support and frequency with large variances only in the atomic amplitude. However, these differences in magnitude can be explained by a reduction in power after filtering. Additional significant atoms can be observed in the noisy decomposition that have time centres significantly distanced in time from the most significant atom. These atoms also often demonstrate much larger time supports than other atoms in the same decomposition and typically have very low or high frequency carrier waves. Heuristic methods could be developed in order to determine more precisely which de-composition atoms correspond to noise signals and those which are atoms characterizing the true percussion event. If such a method were tractable, this could provide noise filtering as part of the classification algorithm itself removing the need for additional signal processing steps and providing for a more efficient system. The spectral content of the inherent noise can be seen in Fig. 10. It is very likely that there is harmonic content within the noise itself based on this information. If this is indeed the case, Gabor atoms will likely match to various portions of the pre and post signal windows.

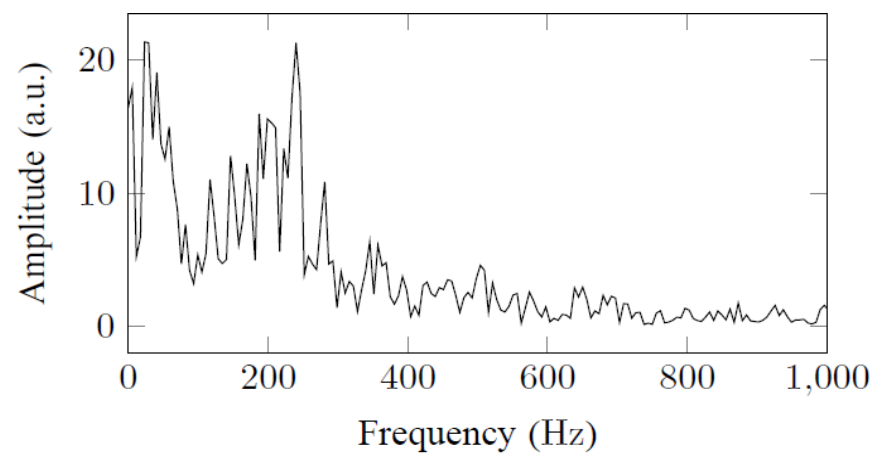

Fig. 10. Inherent noise spectrum sample from percussion signal database. Noise content may contain harmonic terms which could be filtered using Gabor atoms during the MP process rather than pre-process noise filtering.

\subsection{Reconstruction Errors}

The original clean signal $x$ was compared to the reconstruction $\hat{x}$ to determine the percent error.

$$
\text { Percentage Error }=\sqrt{\frac{\sum_{i=1}^{n}(x-\widehat{x})^{2}}{\sum_{i=1}^{n} x^{2}}} \times 100
$$

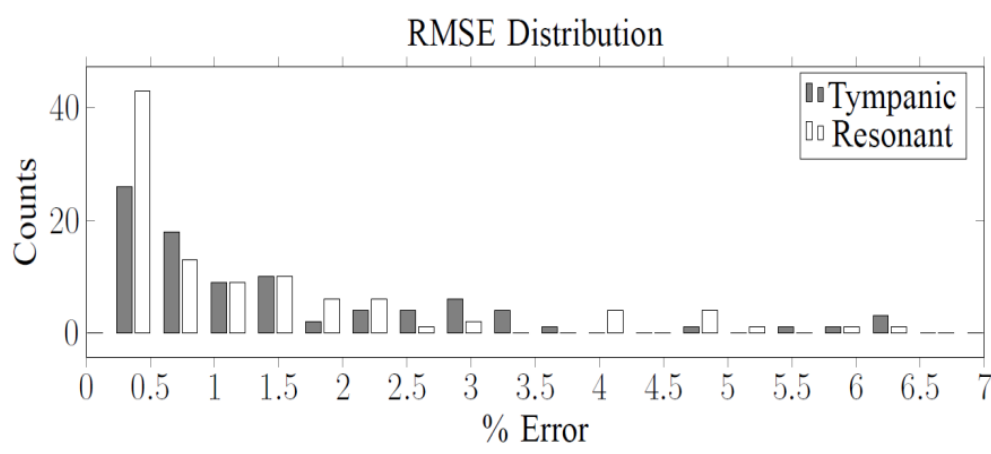

Fig. 11. Histograms of the RMSE comparing the reconstruction to the original percussion signals. Tympanic signals are in dark and resonant signals in white. 
Overall, the entire dataset had an error $1.44 \pm 1.43 \%$ with higher errors correlating to lower initial SNRs. These error values are similar to those found in previous investigations [8]. The error distribution can be seen in Fig. 11.

While both signal classes exhibit similar behaviour, resonant percussions RMSE values tend to be slightly closer to zero than tympanic decompositions. This is likely due to the more localized nature of the signal which will require a narrow time support allowing for a potentially sparser representation if the atoms capture the behaviour of the signal within their window.

\section{Conclusion}

Matching pursuit was examined as a method of decomposing medical percussions and its results compared to previous studies [7], [8]. Dictionary selection criteria were discussed including atom classes and parameter space sizes. Decomposition results were analysed in terms of their errors, their convergence behaviour and the performance of MP under various noise conditions was discussed. The MP decomposition yields a sparse signal representation that can be realized with $O(n \log n)$ complexity allowing for efficient implementations that can be easily adapted to real time applications. MP may also provide robust noise reduction and onset detection strategies. Pursuits are not restricted to the percussion duration and atomic matches made from pre and post stage windows may provide additional information in order to easily localize the signal and to extract external audio sources from the recorded information. In this regard, MP provides a type of signal signature that may prove to be more informative than decompositions simply in terms of harmonic signal components. Considering all these factors, it can be seen that matching pursuit is a robust decomposition technique that is computationally efficient and capable of yielding sparse representations that are on the same order as other methods.

\section{Acknowledgment}

This work was possible due to the financial support of the Office of the Naval Research (USA). The authors would like to express sincere gratitude to the personnel of the Tessonics Corp. and the Department of Physics at the University of Windsor".

\section{References}

[1] Yernault, J. C., \& Bohadana, A. B. (1995). Chest percussion. Eur. Respir. J., 8, 1756-1760.

[2] Mansy, H. A., Royston, T. J., \& Sandler, R. H. (2002). Use of abdominal percussion for pneumoperitoneum detection. Med. Biol. Eng. Comput., 40(4), 439-446.

[3] Hudson, E. D. (1887). A Manual of the Physical Diagnosis of Thoracic Disease. William Wood \& Company, New York.

[4] Bohadana, A. B., \& Kraman, S. S. (1989). Transmission of sound generated by sternal percussion. J. Appl. Physiol., 66(1), 273-277.

[5] Murray, A., \& Neilson, J. M. M. (1975). Diagnostic percussion sounds: 1. A qualitative analysis. Med. Biol. Eng., 13(1), 19-28.

[6] Pantea, M. A., Maev, R. G., Malyarenko, E. V., \& Baylor, A. E. (2012). A physical approach to the automated classification of clinical percussion sounds. J. Acoust. Soc. Am., 131, 608-619.

[7] Bhuiyan, M., Malyarenko, E. V., Pantea, M. A., \& Maev, R. G. (2012). Estimating the parameters of audible clinical percussion signals by fitting exponentially damped harmonics. J. Acoust. Soc. Am., 131(6), 4690-4698.

[8] Bhuiyan, M., Malyarenko, E. V., Pantea, M. A., \& Seviaryn, F. M. (2013). Advantages and limitations of 
using matrix pencil method for the modal analysis of medical percussion signals. IEEE T. Bio-med. Eng., $60(2), 417-426$.

[9] Goodwin, M. M., \& Vetterli, M. (1999). Matching pursuit and atomic signal models based on recursive filter banks. IEEE T. Signal Proces., 47(7), 1890-1902.

[10] Trefethen, L. N., \& Bau, D. (1997). Numerical linear algebra. Society for Industrial and Applied Mathematics, 5, 234-239.

[11] Mallat, S. G., \& Zhang, Z. (1993). Matching pursuits with time-frequency dictionaries. IEEE T. Signal Proces., 41(12), 3397-3415.

[12] I. Daubechies. (1988). Time-frequency localization operators: A geometric phase space approach. IEEE T. Inform. Theory, 34, 605-612.

[13] Coifman, R. R., \& Wickerhauser, M. V. (1992). Entropy-based algorithms for best basis selection. IEEE T. Inform. Theory, 38(2), 713-718.

[14] Krim, H., Tucker, D., Mallat, S., \& Donoho, D. (1999). On Denoising and best signal representation. IEEE T. Inform. Theory, 45(7), 2225-2238.

[15] Chen, S. S., Donoho, D. L., \& Saunders, M. A. (1998). Atomic decomposition by basis pursuit. SIAM J. Sci. Comput., 20(1), 33-61.

[16] Pati, Y. C., Rezaiifar, R., \& Krishnaprasad, P. S. (1993). Orthogonal matching pursuit: Recursive function approximation with applications to wavelet decomposition. Proceedings 27th Asilomar Conference on Signals, Systems \& Computers (pp. 40-44).

[17] Tropp, J. A., \& Gilbert, A. C. (2007). Signal recovery from random measurements via orthogonal matching pursuit. IEEE T. Inform. Theory, 53(12), 4655-4666.

[18] Donoho, D. L., Tsaig, Y., Drori, I., \& Starck, J. (2012). Sparse solution of underdetermined systems of linear equations by stagewise orthogonal matching pursuit. IEEE T. Inform. Theory, 58(2), 1094-1121.

[19] Vera-Candeas, P., Ruiz-Reyes, N., Rosa-Zurera, M., Martinez-Munoz, D., \& Lopez-Ferreras, F. (2004). Transient modeling by matching pursuits with a wavelet dictionary for parametric audio coding. IEEE Signal Proc. Let., 11(3), 349-352.

[20] Neff, R., \& Zakhor. A. (1997). Very low bit-rate video coding based on matching pursuits. IEEE T. Circ. Syst. Vid., 7(1), 158-171.

[21] Ruiz-Reyes, N., Vera-Candeas, P., Curpin-Alonso, J., Mata-Campos, R., \& Cuevas-Martnez J. C. (2005). New matching pursuit-based algorithm for SNR improvement in ultrasonic NDT. NDT\&E Int., 38(6), 453-458.

[22] Ghofrani, S., McLernon, D. C., \& Ayatollahi, A. (2005). On conditional spectral moments of Gaussian and damped sinusoidal atoms in adaptive signal decomposition. Signal Process., 85(10), 1984-1992.

[23] Mallat, S. (1999). A Wavelet Tour of Signal Processing. San Diego: Academic Press.

[24] Krstulovic, S., \& Gribonval, R. (2006). MPTK: Matching pursuit made tractable. Proceedings of Conf. Acoust. Speech Signal Process: Vol. 3 (pp. 496-499).

[25] Plapous, C., Marro, C., \& Scalart, P. (2006). Improved signal-to-noise ratio estimation for speech enhancement. IEEE Audio, Speech, and Language Processing, 14(6), 2098-2108.

Jeff Dech received his B.Sc. Hon. degree in physics \& high technology at the University of Windsor, Windsor, ON in 2012. He is currently a masters student in physics at the University of Windsor, studying in atomic, molecular \& optical physics under the guidance of Dr. J. W. McConkey. He has previously worked in the fields of acoustic and digital signal processing with the Institute for Diagnostic Imaging Research. 


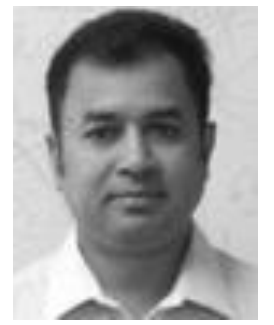

Moinuddin Bhuiyan received the B.Sc. degree in electrical and electronic engineering from Chittagong University of Engineering and Technology (CUET), Chittagong, Bangladesh, in 1994, and the M.Sc. degree in electrical and computer engineering from Ryerson University, Toronto, Canada, in 2006. He received his Ph.D. in electrical engineering from the University of Windsor in 2013. After completion of his degree he was appointed as a postdoc scholar fellow at Institute for Diagnostic Imaging Research at the University of Windsor, Windsor, Canada. Moinuddin Bhuiyan's research interests include signal and image processing, statistical signal processing, pattern recognition and their applications.

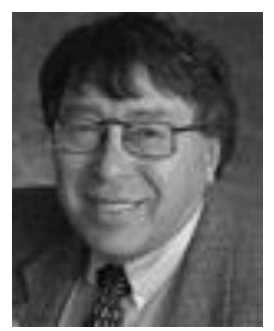

Roman Gr. Maev received the B.Sc. combined with M.Sc. degrees in theoretical solid state and nuclear physics from Moscow Physical Engineering Institute, Moscow, Russia, 1969. In 1973, he received the Ph.D. degree in theory of photo-semiconductor physics from Physical PN Lebedev Institute of the USSR, Academy of Sciences, Moscow, Russia. In 2002, he received the D.Sc. degree from the Russian Academy of Sciences, Moscow, for methods of acoustic microscopy for investigation of microstructure, physical and chemical properties of materials. In 2005, he was a full professor in physics from the Russian Federation Government in scientific equipment and methods for experimental physics, he is the founding director general of the Institute for Diagnostic Imaging Research, University of Windsor, Windsor, ON, Canada, which is a multidisciplinary, collaborative research and innovation consortium. He is also a full faculty professor in the Department of Physics, University of Windsor, Windsor, and in 2007 he was granted the title of distinguished university professor. In 2004, based on an initiative by chryslers, a new Canadian start-up high-tech Company "Tessonics Corporation" was created with its main mission for the commercialization of the research products developed by Maev's R\&D team at the University of Windsor. $\mathrm{He}$ is providing an important leading technical role in this new enterprise. The extraordinarily diverse range of disciplines encompassed by him includes theoretical fundamentals of physical acoustics, research in ultrasonic and nonlinear acoustical imaging, nano-structural properties of advanced materials, and its analysis. He is an author of 4 monographs, an editor and co-editor of 9 books, has published more than 440 per-reviewed items, including 113 articles in leading international journals, 287 referred conferences proceedings, and holds 29 international patents. Dr. Maev has been a member of the Canada-Russia Intergovernmental Economical Commission and Canada-Russia Business Council since 2001 and in 2008 he was appointed as an honorary consul of the Russian Federation in Canada (Windsor, ON). He became the DaimlerChrysler/NSERC industrial research chair in 2002 and the Fiat/Chrysler/NSERC industrial research chair in advanced materials characterization in 2008. He is a fellow of the British Institute of Non-Destructive Testing. 